TAIWANESE JOURNAL OF MATHEMATICS

Vol. 12, No. 5, pp. 1061-1066, August 2008

This paper is available online at http://www.tjm.nsysu.edu.tw/

\title{
A TAUBERIAN THEOREM FOR UNIFORMLY WEAKLY CONVERGENCE AND ITS APPLICATION TO FOURIER SERIES
}

\author{
Chang-Pao Chen and Meng-Kuang Kuo
}

\begin{abstract}
In 1995, S. Mercourakis introduced the concept of uniformly weakly convergent sequences and characterized such sequences as those with the property that any of its subsequences is Cesàro-summable. In this paper, we present a Tauberian theorem for such kind of convergence. As a consequence, we prove that the uniformly pointwise convergence and the uniform convergence of a sequence of complex-valued functions coincide under a suitable Tauberian condition. This result affirmatively answers a question raised by S. Mercourakis concerning the Fourier series of a continuous function on the circle group $T$. In this paper, a result of Banach type is also established for uniformly weakly convergent sequences. Our result generalizes the work of Mercourakis.
\end{abstract}

\section{INTRODUCTION}

Let $(X,\|\cdot\|)$ be a Banach space and $f_{n}, f \in X$. In the theory of mathematical analysis, pointwise convergence and uniform convergence are two important concepts in the literature (cf. [7] and [8]). They are exhaustively studied in many aspects, e.g., in the metric theory of functions (cf. [9]) and in Fourier series (cf. [1] and [11]). The notion of pointwise convergence was extended to the Banach space theory in the following setting for a long time (cf. [4]). We say that $f_{n} \rightarrow f$ weakly in $X$ if $\lambda\left(f_{n}\right) \rightarrow \lambda(f)$ for every $\lambda \in X^{*}$, where $X^{*}$ denotes the dual space of $X$ consisting of all continuous linear functionals $\lambda$ on $X$. This concept has shown its importance in the study of the classical Banach spaces, e.g., in the study of the Banach-Saks property (cf. [3, Chapter VII]). Corresponding to the uniform

Received May 6, 2006, accepted July 5, 2006.

Communicated by Sen-Yen Shaw.

2000 Mathematics Subject Classification: 40A30, 40E05, 40 G05.

Key words and phrases: Uniformly weakly convergence, Tauberian conditions, Uniform convergence of Fourier series.

This work is supported by the National Science Council, Taipei, ROC, under Grant NSC 96-2115-M364-003-MY3. 
convergence, Mercourakis [6, Definition 2.1] introduced the concept of uniformly weakly convergence, which is defined as follows. We say that $f_{n} \longrightarrow f$ uniformly weakly in $X$ if for each $\varepsilon>0$ there exists a natural number $N(\varepsilon)$ such that

$$
\sharp\left(\left\{n:\left|\lambda\left(f_{n}\right)-\lambda(f)\right| \geq \varepsilon\right\}\right) \leq N(\varepsilon) \quad\left(\lambda \in X^{*} ;\|\lambda\| \leq 1\right) .
$$

Here the notation $\sharp$ denotes the cardinality of a set. In [6, Theorem 2.6], Mercourakis characterized uniformly weakly convergent sequences as those obeying the property that any of its subsequences is Cesàro-summable in $X$. He also proved that $f_{n} \longrightarrow f$ uniformly weakly in $X$ if and only if

$$
\lim _{N \rightarrow \infty}\left\{\sup _{k_{1}<\cdots<k_{N}}\left\|\frac{1}{N} \sum_{i=1}^{N} f_{k_{i}}-f\right\|\right\}=0 .
$$

These results led Mercourakis to characterize the Banach-Saks and the weak BanachSaks properties from the viewpoint of uniformly weakly convergence (cf. [6, Theorems 2.9 and 2.10]).

For $f_{n} \in C(\Omega)$, where $\Omega$ is a given compact Hausdorff space, we have the following implications:

$$
\begin{aligned}
\|\cdot\|_{\infty}-\text { convergence } & \Longrightarrow \text { uniformly weakly convergence } \\
& \Longrightarrow \text { weak convergence } \\
& \Longrightarrow \text { pointwise convergence. }
\end{aligned}
$$

It is known (see, for example, [3] and [6, p.91]) that the converse implications in (1.3) are false, in general. In [3, p. 66, Theorem 1], Banach proved that $f_{n} \rightarrow f$ weakly in $C(\Omega)$ if and only if $\sup _{n}\left\|f_{n}\right\|_{\infty}<\infty$ and $f_{n} \rightarrow f$ pointwise on $\Omega$. This result has been extended by Mercourakis to uniformly weakly convergence (see [6, Proposition 2.2]). He proved that for a given uniformly bounded sequence, $f_{n} \rightarrow f$ uniformly weakly in $C(\Omega)$ if and only if $f_{n} \rightarrow f$ uniformly pointwise on $\Omega$, that is, for each $\epsilon>0$ there exists a natural number $N(\varepsilon)$ such that

$$
\sharp\left(\left\{n:\left|f_{n}(\gamma)-f(\gamma)\right| \geq \varepsilon\right\}\right) \leq N(\varepsilon) \quad(\gamma \in \Omega) .
$$

Mercourakis's result only deals with uniformly bounded sequences. We shall prove in Lemma 2.2 that it can be extended to the general case in a form of Banach type. We shall see its application later. As for the implication from weak convergence to uniformly weakly convergence, this part involves the Banach-Saks or the weak Banach-Saks property. We refer the readers to [3, pp. 109-113] and [6, pp. 101103] for details.

In [6, p.103], Mercourakis asked a question of the implication from uniformly weakly convergence to norm convergence. His question reads as follows. Let 
$s_{n}(f ; t)$ denote the $n$th partial sum of the Fourier series of $f \in C(T)$, where $T=$ $[-\pi, \pi]$. Suppose that $\left\{s_{n}(f)\right\}_{n=0}^{\infty}$ is uniformly bounded and converges uniformly pointwise on $T$ to $f$. Does then $s_{n}(f)$ converge uniformly on $T$ to $f$ ? This question is still open. The purpose of this paper is to answer this question affirmatively. To do so, we first establish a Tauberian theorem for uniformly weakly convergence (see Theorem 2.1). More precisely, we shall prove that under (1.5), uniformly weakly convergence implies norm convergence:

$$
\lim _{n \rightarrow \infty}\left\|f_{n+1}-f_{n}\right\|=0 .
$$

Such a condition is known as a Tauberian condition and the corresponding result is called a Tauberian theorem (see [2] and [5] for the definitions). With the help of Lemma 2.2, we deduce the second form of the aforementioned Tauberian theorem for $X=\ell^{\infty}(\Gamma)$ or $C(\Omega)$, in which the concept of uniformly pointwise convergence is involved. This result says that the $\|\cdot\|_{\infty}$ convergence coincides with the uniformly pointwise convergence under condition (1.5) (see Theorem 2.4). For $f_{n}=s_{n}(f ; t)$, condition (1.5) is automatically satisfied and so Theorem 2.4 answers the question of Mercourakis affirmatively.

\section{MAIN Results}

The following result gives a Tauberian theorem for uniformly weakly convergence.

Theorem 2.1. Let $\left\{f_{n}\right\}_{n=0}^{\infty}$ be a sequence in a Banach space $(X,\|\cdot\|)$ and $f \in X$. Then $f_{n} \rightarrow f$ in $X$ if and only if $f_{n} \rightarrow f$ uniformly weakly in $X$ and (1.5) is satisfied.

Proof. The "only if" part follows from the definitions. We prove the converse. Assume that $f_{n} \rightarrow f$ uniformly weakly in $X$ and (1.5) is satisfied. By the uniform boundedness theorem (cf. [10, p.68]), we know that $\left\{\left\|f_{n}\right\|\right\}_{n=0}^{\infty}$ is bounded. It follows from [6, Theorem 2.6] that (1.2) is true. We have

$$
\begin{aligned}
\left\|f_{n}-f\right\| & \leq\left\|\frac{1}{N} \sum_{k=n}^{n+N-1} f_{k}-f\right\|+\frac{1}{N} \sum_{k=n}^{n+N-1}\left\|f_{k}-f_{n}\right\| \\
& \leq \sup _{k_{1}<\cdots<k_{N}}\left\|\frac{1}{N} \sum_{i=1}^{N} f_{k_{i}}-f\right\|+(N-1)\left\{\sup _{k \geq n}\left\|f_{k+1}-f_{k}\right\|\right\} .
\end{aligned}
$$

By (1.2) and (1.5), we conclude that $f_{n} \rightarrow f$ in $X$. This completes the proof. 
The condition (1.5) can not be removed from Theorem 2.1. For instance, consider $X=c_{0}(\mathbb{N})$ (or $\ell^{2}(\mathbb{N})$ ) and $f_{n}=e_{n}$, where $\mathbb{N}$ denotes the set of all nonnegative integers and $e_{n}$ is the sequence with 1 at the $n$th position and 0 otherwise. We have that $f_{n} \rightarrow 0$ uniformly weakly in $X$ but $f_{n} \nrightarrow 0$ in $X$. Even for the case $X=C[0,1],(1.5)$ is still necessary. A counterexample is given by

$$
f_{n}(x)= \begin{cases}2(n+1)(n+2)\left(x-\frac{1}{n+2}\right) & \text { on }\left(\frac{1}{n+2}, \frac{2 n+3}{2(n+1)(n+2)}\right), \\ -2(n+1)(n+2)\left(x-\frac{1}{n+1}\right) & \text { on }\left[\frac{2 n+3}{2(n+1)(n+2)}, \frac{1}{n+1}\right), \\ 0 & \text { otherwise. }\end{cases}
$$

In the following, we assume that $\Gamma$ is a nonempty set and $\Omega$ is a compact Hausdorff space. In order to get the second form of Theorem 2.1, we need the following generalization of [6, Proposition 2.2]. This is a result of Banach type.

Lemma 2.2. $\quad f_{n} \rightarrow f$ uniformly weakly in $\ell^{\infty}(\Gamma)$ (respectively, $C(\Omega)$ ) if and only if $\sup _{n}\left\|f_{n}\right\|_{\infty}<\infty$ and $f_{n} \rightarrow f$ uniformly pointwise on $\Gamma$ (respectively, $\Omega$ ).

Proof. We know that any uniformly weakly convergent sequence is bounded, so we can easily deduce the "only if" part by using the fact that $(1.1) \Longrightarrow(1.4)$. The if part of the case $C(\Omega)$ follows from [6, Proposition 2.2]. As for the case $\ell^{\infty}(\Gamma)$, by [6, Theorems $\left.1.8 \& 2.6\right]$, we find that for uniformly bounded sequences, uniformly weakly convergence $\Longleftrightarrow(1.2) \Longleftrightarrow$ uniformly pointwise convergence. This leads us to the conclusion.

The condition $\sup _{n}\left\|f_{n}\right\|_{\infty}<\infty$ in Lemma 2.2 is necessary. The following example displays this fact: let $f_{n}(\gamma)=n+1$ for $\gamma=1 /(n+1)$ and 0 otherwise. Then $f_{n} \rightarrow 0$ uniformly pointwise on $[0,1]$, but $f_{n} \nrightarrow 0$ uniformly weakly in $\ell^{\infty}([0,1])$.

For uniformly pointwise convergent sequences, we show below that the condition $\sup _{n}\left\|f_{n}\right\|_{\infty}<\infty$ can be derived from (1.5).

Lemma 2.3. Let $X=\ell^{\infty}(\Gamma)$ or $C(\Omega)$ and $f_{n}, f \in X$. If $f_{n} \rightarrow f$ uniformly pointwise and (1.5) holds, then $\sup _{n}\left\|f_{n}\right\|_{\infty}<\infty$.

Proof. We prove the case $X=\ell^{\infty}(\Gamma)$ and leave $X=C(\Omega)$ to the readers. Without loss of generality, we assume $f=0$. Since $f_{n} \rightarrow 0$ uniformly pointwise on $\Gamma$, there exists a positive integer $N$ such that $\sharp\left(\left\{n:\left|f_{n}(\gamma)\right| \geq 1\right\}\right) \leq N$ for all $\gamma \in \Gamma$. This implies that for any $n$ and any $\gamma$, one of $\left|f_{n}(\gamma)\right|,\left|f_{n+1}(\gamma)\right|, \cdots,\left|f_{n+N}(\gamma)\right|$ is less than 1 , say $\left|f_{m}(\gamma)\right|$, and so

$$
\left|f_{n}(\gamma)\right| \leq \sum_{k=n}^{m-1}\left|f_{k+1}(\gamma)-f_{k}(\gamma)\right|+\left|f_{m}(\gamma)\right| \leq N\left(\sup _{k \geq 0}\left\|f_{k+1}-f_{k}\right\|_{\infty}\right)+1
$$


Taking supremum over $n$ and $\gamma$ gives $\sup _{n}\left\|f_{n}\right\|_{\infty}<\infty$. This completes the proof.

Putting Theorem 2.1 and Lemmas 2.2-2.3 together, we get the second form of Theorem 2.1 for $X=\ell^{\infty}(\Gamma)$ or $C(\Omega)$.

Theorem 2.4. Let $X=\ell^{\infty}(\Gamma)$ or $C(\Omega)$ and $f_{n}, f \in X$. Then $f_{n} \rightarrow f$ in $X$ if and only if $f_{n} \rightarrow f$ uniformly pointwise and (1.5) holds.

For $f \in C(T)$, we know that $\lim _{n \rightarrow \infty}\left\|s_{n+1}(f)-s_{n}(f)\right\|_{\infty}=0$. This can be proved by using the Riemann-Lebesgue theorem (see [11, Vol. I, p.45]). Hence, the condition (1.5) with $f_{n}=s_{n}(f)$ holds. As a consequence of Theorem 2.4, we conclude that $s_{n}(f) \rightarrow f$ uniformly on $T$ if and only if $s_{n}(f) \rightarrow f$ uniformly pointwise on $T$. This answers the question of Mercourakis affirmatively. Moreover, the condition of uniformly boundedness required there for $s_{n}(f), n \geq 0$, is not necessary.

\section{REFERENCES}

1. N. K. Bary, A Treatise on Trigonometric Series, Vols. I \& II. Pergamon Press, New York, 1964.

2. Chang-Pao Chen and Jui-Ming Hsu, Tauberian theorems for weighted means of double sequences, Anal. Math., 26 (2000), 243-262.

3. J. Diestel, Sequences and Series in Banach Spaces, Graduate Texts in Math. 92, Springer-Verlag, 1984.

4. N. Dunford and J. T. Schwartz, Linear Operators, Part I: General Theory, Interscience, New York, 1958.

5. G. H. Hardy, Divergent Series, Oxford University Press, New York, 1949.

6. S. Mercourakis, On Cesàro summable sequences of continuous functions, Mathematika 42(1995), no. 1, 87-104.

7. W. Rudin, Principles of Mathematical Analysis, 3rd ed., McGraw-Hill, New York, 1976.

8. E. C. Titchmarsh, The Theory of Functions, 2nd ed., Oxford University Press, London, 1939; Russian transl. Nauka Moscow, 1980.

9. P. L. Ul'yanov, The metric theory of functions. (Russian) English transl in Proc. Steklov Inst. Math. 1990, No. 1, 199-244. Probability theory, function theory, mechanics (Russian). Trudy Mat. Inst. Steklov., 182 (1988), 180-223. 
10. K. Yosida, Functional Analysis, 2nd ed., Springer, Berlin-Heidelberg-New York, 1968.

11. A. Zygmund, Trigonometric Series, Vols. I \& II combined, 3rd ed., Cambridge University Press, New York, 2002.

Chang-Pao Chen and Meng-Kuang Kuo

Department of Applied Mathematics,

Hsuan Chuang University,

Hsinchu 300, Taiwan, R.O.C.

E-mail: cpchen@wmail.hcu.edu.tw 